\title{
In vitro anti-malarial interaction and gametocytocidal activity of cryptolepine
}

\author{
Arnold Donkor Forkuo 1*, Charles Ansah', Kwesi Boadu Mensah', Kofi Annan', Ben Gyan², Anjo Theron³, \\ Dalu Mancama ${ }^{3}$ and Colin W. Wright ${ }^{4}$
}

\begin{abstract}
Background: Discovery of novel gametocytocidal molecules is a major pharmacological strategy in the elimination and eradication of malaria. The high patronage of the aqueous root extract of the popular West African anti-malarial plant Cryptolepis sanguinolenta (Periplocaceae) in traditional and hospital settings in Ghana has directed this study investigating the gametocytocidal activity of the plant and its major alkaloid, cryptolepine. This study also investigates the anti-malarial interaction of cryptolepine with standard anti-malarials, as the search for new anti-malarial combinations continues.
\end{abstract}

Methods: The resazurin-based assay was employed in evaluating the gametocytocidal properties of $C$. sanguinolenta and cryptolepine against the late stage (IVN) gametocytes of Plasmodium falciparum (NF54). A fixed ratio method based on the SYBR Green I fluorescence-based assay was used to build isobolograms from a combination of cryptolepine with four standard anti-malarial drugs in vitro using the chloroquine sensitive strain 3D7.

Results: Cryptolepis sanguinolenta $\left(\mathrm{IC}_{50}=49.65 \mathrm{nM}\right)$ and its major alkaloid, cryptolepine $\left(\mathrm{IC}_{50}=1965 \mathrm{nM}\right)$, showed high inhibitory activity against the late stage gametocytes of P. falciparum (NF54). In the interaction assays in asexual stage, cryptolepine showed an additive effect with both lumefantrine and chloroquine with mean $\Sigma \mathrm{FIC}_{50} \mathrm{~S}$ of $1.017 \pm 0.06$ and $1.465 \pm 0.17$, respectively. Cryptolepine combination with amodiaquine at therapeutically relevant concentration ratios showed a synergistic effect (mean $\Sigma F C_{50}=0.287 \pm 0.10$ ) whereas an antagonistic activity (mean $\left.\Sigma \mathrm{FIC}_{50}=4.182 \pm 0.99\right)$ was seen with mefloquine.

Conclusions: The findings of this study shed light on the high gametocytocidal properties of C. sanguinolenta and cryptolepine attributing their potent anti-malarial activity mainly to their effect on both the sexual and asexual stages of the parasite. Amodiaquine is a potential drug partner for cryptolepine in the development of novel fixed dose combinations.

Keywords: Gametocytocidal, Malaria, Anti-malarial drug combinations, Cryptolepis sanguinolenta, Cryptolepine

\section{Background}

Malaria still remains a disease of public health importance with an estimated 3.3 billion people at risk globally and 214 million new cases with 438,000 deaths reported in 2015. The sub-Saharan region of Africa accounts for an estimated $90 \%$ of all deaths due to malaria, with children under 5 years of age accounting for $78 \%$ of these [1].

\footnotetext{
*Correspondence: forkuoarnold@yahoo.com

1 Department of Pharmacology, Faculty of Pharmacy and Pharmaceutical

Sciences, College of Health Sciences, Kwame Nkrumah University

of Science and Technology, Kumasi, Ghana

Full list of author information is available at the end of the article
}

Despite the successes seen in the approach to reduce the number of deaths associated with malaria, this disease still remains a grave public health problem. The majority of the currently used anti-malarial drugs were intended to target the symptom-causing pathogenic blood stages in man and to contend with the continuous risk of drug resistance [2]. Nonetheless, to achieve the global objective of malaria eradication, medicines with activity against parasite transmission [3] and the hepatic stages responsible for disease relapse also need to be developed. The reported development of Plasmodium falciparum resistance to the most potent anti-malarial 
agents (the artemisinins) in the Thai-Cambodia border puts the progress achieved under serious threat $[4,5]$. This scenario requires the urgent need to accelerate the discovery and development of novel anti-malarial leads and combinations that have activity against both the sexual and asexual stages of the parasite.

For this reason, the popular West African anti-malarial plant Cryptolepis sanguinolenta (Periplocaceae) and it major alkaloid, cryptolepine were assessed against the transmissible stages of the human malaria parasite. The aqueous root extract of $C$. sanguinolenta has been used for decades in West Africa for the treatment of malaria [6] and currently, several herbal preparations registered for use in Ghanaian orthodox clinics contain the aqueous root extract of the plant. The wide usage of the plant may be attributed to the safety and high cure rate of a tea bag formulation (Phyto-laria) of the plant against chloroquine resistant strains of $P$. falciparum in human clinical trials [7], supporting their usage as alternative tools to the standard anti-malarial interventions or as complementary treatments in the absence of standard anti-malarial drugs. Cryptolepine, the major indoloquinoline alkaloid isolated from the plant has the most potent antiplasmodial activity [8] and has been shown to exhibit potent in vitro activities against both chloroquine-sensitive and chloroquine-resistant $P$. falciparum [9].

With lessons learnt from the widely used artemisinin derivatives, thus their isolation from the medicinal plant Artemisia annua, directed the exploration of the popularly used anti-malarial plant, $C$. sanguinolenta and its major alkaloid, cryptolepine for possible gametocytocidal effects on P. falciparum. The in vitro anti-malarial interaction of cryptolepine with chloroquine, lumefantrine, amodiaquine and mefloquine (Fig. 1) were also ascertained in this study.

\section{Methods}

\section{Plant material}

The sun-dried cut roots of C. sanguinolenta used in this study were collected at the Centre for Scientific Research into Plant Medicine (CSRPM), Mampong-Akwapim, Ghana in August 2012 and were identified by the Plant Development Centre of the Institution. Its authenticity was confirmed by Dr. Kofi Annan of the Department of Pharmacognosy, KNUST and subsequently compared to a voucher specimen KNUST/HM1/2008/L056 at the herbarium of the Department of Pharmacognosy/Herbal Medicine, College of Health Sciences.

The powdered roots $(650 \mathrm{~g})$ were boiled for $30 \mathrm{~min}$ with $5 \mathrm{~L}$ of distilled water, decanted and filtered. The filtrate was freeze dried to obtain a freeze-dried sample of the crude extract (yield $=11.08 \% \mathrm{w} / \mathrm{w}$ ) referred to as cryptolepis (CPS). Cryptolepis was reconstituted in distilled water prior to use in the gametocytocidal assay.

\section{Drugs/chemicals}

Lumefantrine, chloroquine, mefloquine and amodiaquine were purchased from Sigma-Aldrich (St. Louis, MO, USA). Gentamicin was obtained from Invitrogen Life Technologies Inc. (Carlsbad, CA, USA). RPMI1640 medium, streptomycin/penicillin, L-glutamine and HEPES were obtained from Gibco BRL Life Technologies (Grand Island, NY, USA). Cryptolepine hydrochloride (purity 98.9\%) was isolated from the root of C. sanguinolenta as described by Kuntworbe et al. [10].

In vitro cultivation of asexual stage Plasmodium falciparum The in vitro cultivation method of $P$. falciparum drugsensitive strain NF54 was adapted from Reader et al. [11]. Parasite cultures were maintained in human erythrocytes (5\% haematocrit, A rhesus positive) suspended in complete parasite medium (CPM) (RPMI 1640 medium supplemented with $25 \mathrm{mM}$ HEPES, $0.2 \%$ D-glucose, $24 \mu \mathrm{g} /$ $\mathrm{mL}$ gentamicin, $0.2 \%$ sodium bicarbonate $(\mathrm{pH}=7.3)$, $200 \mu \mathrm{M}$ hypoxanthine with $10 \%$ human serum (A rhesus positive) and flushed with $90 \% \mathrm{~N}_{2}, 5 \% \mathrm{O}_{2}$, and $5 \% \mathrm{CO}_{2}$ in humidified modular chambers at $37{ }^{\circ} \mathrm{C}$. Parasite medium was changed daily and fresh CPM introduced. Parasitaemia of Giemsa-stained slides were monitored daily with light microscopy.

\section{Induction of gametocytogenesis and maintenance of gametocyte cultures}

Combined conditions of low haematocrit and nutrient starvation were employed in the induction of gametocytogenesis. To trigger gametocytogenesis, asexual cultures of $6-10 \%$ parasitaemia were diluted to $0.5 \%$ parasitaemia at $6 \%$ haematocrit and then introduced into a glucose-free medium. The medium was kept at $37{ }^{\circ} \mathrm{C}$ gassed with $90 \% \mathrm{~N}_{2}, 5 \% \mathrm{O}_{2}$, and $5 \% \mathrm{CO}_{2}$ without shaking. The medium was changed daily. After $72 \mathrm{~h}$, the haematocrit was dropped to $3 \%$ (day 0). Gametocytogenesis was monitored daily before medium (glucose-free) was changed. To eliminate residual asexual parasites, cultures were treated on days $6-9$ by continuous treatment with $50 \mathrm{mM} \mathrm{N}$-acetyl glucosamine (NAG). The medium was then fortified with $0.2 \%$ glucose from day 10 onwards. The gametocyte levels were monitored daily by microscopy until they were predominantly $(>90 \%)$ stage $\mathrm{V}$ and these gametocytes were employed in the resazurin-based assay.

\section{Gametocyte viability assays}

The in vitro gametocytocidal activities of cryptolepine and the aqueous root extract of $C$. sanguinolenta were 
<smiles>OC(c1cc(C(F)(F)F)nc2c(C(F)(F)F)cccc12)C1CCCCN1</smiles>

C<smiles>CCN(CC)CCC[C@H](C)Nc1ccnc2cc(Cl)ccc12</smiles><smiles>CCN(CC)Cc1cc(Nc2ccnc3cc(Cl)ccc23)ccc1O</smiles><smiles>CCCCN(CCCC)CC(O)c1cc(Cl)cc2c1-c1ccc(Cl)cc1C2=Cc1ccc(Cl)cc1</smiles>

e<smiles></smiles>

Fig. 1 Structure of anti-malarial agents used in the interaction assay. a Mefloquine, $\mathbf{b}$ amodiaquine, $\mathbf{c}$ chloroquine, $\mathbf{d}$ lumefantrine, e crytolepine hydrochloride

measured by assessing gametocyte survival after drug exposure using resazurin-based assay. The resazurinbased assay was based on method described by Tanaka et al. [12]. Eleven drug concentrations of cryptolepine, the aqueous root extract of $C$. sanguinolenta and dihydroartemisinin ranging from $10 \mu \mathrm{M}$ to $1.69 \times 10^{-4} \mu \mathrm{M}$ (threefold serial dilutions) prepared in distilled water were placed in triplicate in a transparent 96-well flat bottom plates. Parasitized RBCs were added to a final concentration of $5 \%$ haematocrit, $2 \%$ gametocytaemia in a total incubation volume of $100 \mu \mathrm{L}$.

Dihydroartemisinin $(10 \mu \mathrm{M})$ was used as a reference standard in the drug assay. The drug plate was placed on a mechanical shaker for $20 \mathrm{~s}$ before being encased in an airtight chamber and gassed for $5 \mathrm{~min}$ with a $5 \% \mathrm{O}_{2}, 5 \% \mathrm{CO}_{2}$ and a balanced $\mathrm{N}_{2}$ mixture. The plates were incubated at $37^{\circ} \mathrm{C}$ for $48 \mathrm{~h}$ after which $10 \mu \mathrm{L}$ of resazurin-based assay reagent was added to each well and the plate was shaken 
for $20 \mathrm{~s}$. The plate was left to incubate for $2 \mathrm{~h}$ and then centrifuged at $120 \times g$ for $1 \mathrm{~min}$. The supernatant $(70 \mu \mathrm{L})$ was transferred to a clean 96 -well plate before being read in a multiwell spectrophotometer (Infinite F500, Tecan, USA) by fluorescence detection at 535/612 nm. Gametocyte survival in each test culture after treatment was calculated relative to the control wells. The experiment was performed in triplicate on two separate occasions.

\section{Antiplasmodial interaction assay In vitro malaria parasite cultivation}

Plasmodium falciparum laboratory strain 3D7 was cultured according to method described by Trager and Jensen [13] with slight modifications as described. Human erythrocytes ( $\mathrm{O}$ rhesus positive) fortified in complete culture medium ( $\mathrm{pH}$ 7.3) served as host cells for parasite maturation. The complete culture medium consisted of filter-sterilized RPMI 1640 solution supplemented with 0.5\% AlbuMAX II, $0.72 \%$ HEPES (N-2-hydroxyethylpiperazine- $N$-2-ethanesulfonic acid) and hypoxanthine buffered with $0.4 \%$ sodium bicarbonate $\left(\mathrm{NaHCO}_{3}\right)$. Gentamicin $(0.005 \mathrm{mg} / \mathrm{mL})$ was added to the final solution.

A controlled experimental environment with a gas supply of $92 \% \mathrm{~N}_{2}, 5 \% \mathrm{CO}_{2}$ and $3 \% \mathrm{O}_{2}$ at $37{ }^{\circ} \mathrm{C}$ was used to grow the parasites in culture flasks. Parasite growth, viability and stages were monitored daily by light microscopy of Giemsa-stained cultures after changing the culture media. Parasitaemia levels were kept between 2 and $8 \%$, with a $5 \%$ haematocrit.

\section{In vitro antiplasmodial interaction assay}

The SYBR Green I-based fluorescence assay was employed to investigate the in vitro anti-malarial interaction between cryptolepine and the four standard anti-malarial agents against $P$. falciparum (chloroquine sensitive, 3D7). Cryptolepine (Drug A, CPE), lumefantrine (LUM), mefloquine (MFQ), chloroquine (CQ) and amodiaquine (AMQ) stock solutions were prepared in ethanol at $1 \mathrm{mM}$. Concentrations ranging from 32.5 to $2080 \mathrm{nM}$ for CPE, 2 to $640 \mathrm{nM}$ for LUM, 3.2 to $83 \mathrm{nM}$ for CQ, 5 to $800 \mathrm{nM}$ for MFQ and 1 to $128 \mathrm{nM}$ for AMQ were used for the interaction assays. A fixed ratio interaction assay as described previously by Fivelman et al. [14] was employed in this assay.

For each assay, fixed drug ratios (4:1, 3:2, 2:3 and 1:4) were prepared in a $10 \mu \mathrm{L}$ volume for cryptolepine (Drug A) and any of the standard agents (Drug B). A twofold serial dilution followed each fixed ratio in a well ensuring the $\mathrm{IC}_{50}$ of each drug alone (5:0 and 0:5) fell approximately at the mid-point of the serial dilution. Parasite culture $(90 \mu \mathrm{L})$ was added to each well to obtain seven desired final concentrations for the assay. The final haematocrit and parasitaemia of the culture media were 2 and $1 \%$, respectively. The assay plates were gassed with a mixture of gases containing $92 \% \mathrm{~N}_{2}, 5 \% \mathrm{CO}_{2}$ and $3 \% \mathrm{O}_{2}$ for $6 \mathrm{~min}$ and carefully arranged in a modular incubator (Billups-Rothenberg Inc, USA). The plates were incubated at $37{ }^{\circ} \mathrm{C}$ for $48 \mathrm{~h}$. Post incubation, the plates were stored at $-30{ }^{\circ} \mathrm{C}$ wrapped in aluminium foil. The plates were thawed and subsequently mixed with $100 \mu \mathrm{L}$ of Malaria SYBR Green 1 fluorescent (MSF) lysis buffer containing SYBR Green. The plates were incubated at room temperature in the dark for an hour and fluorescence data (relative fluorescence unit, RFU) were acquired using a fluorescence multi-well plate reader (Tecan Infinite M200 Pro) with emission and excitation wavelength at 535 and $485 \mathrm{~nm}$, respectively. The experiment was performed in triplicate.

\section{Statistical analysis}

The performance of the gametocyte assay was assessed by estimating the $\mathrm{Z}$ factor statistical parameter described by Zhang et al. [15]. Gametocyte viability was calculated using the relation below

$$
\begin{aligned}
& \text { \% Parasite viability }
\end{aligned}
$$

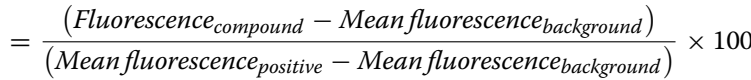

The $\mathrm{IC}_{50}$ (50\% inhibitory concentration) served as a measure of anti-malarial activity and was determined by plotting and analysing dose-response curves using GraphPad Prism (GraphPad 6 Software, San Diego, USA). The assessment of drug interaction was based on calculating the sum of the fractional inhibitory concentration $\left(\Sigma \mathrm{FIC}_{50} \mathrm{~s}\right)$ at the given effective concentration by the formula below

$$
\begin{aligned}
\sum \text { FIC }_{50}= & \left(\frac{I C_{50} \text { of cryptolepine in combination }}{I C_{50} \text { of cryptolepine alone }}\right) \\
& +\left(\frac{I C_{50} \text { of Drug B in combination }}{I C_{50} \text { of Drug B alone }}\right)
\end{aligned}
$$

The nature of interaction was explained using $\Sigma \mathrm{FIC}_{50} \mathrm{~s}$. Values $<0.8$ denote synergism, 0.8-1.4 denotes additive interaction, and $\geq 1.4$ denotes antagonism [16]. The overall nature of the anti-malarial interaction was based on the mean $\Sigma \mathrm{FIC}_{50} \mathrm{~s}$.

A Microsoft Excel datasheet was used to calculate percentage inhibition in relation to the control results. Figures were made using GraphPad Prism for Windows version 6.0 (GraphPad Software, San Diego, CA, USA). 


\section{Results}

\section{Gametocyte viability assay}

Data from the late stage gametocyte assay indicates a preferential inhibition of the viability of mature sexualstage parasites by the aqueous extract of $C$. sanguinolenta and its major alkaloid cryptolepine. The reduction in gametocytes was in a dose-dependent fashion in all drug treatments and statistically significant. $\mathrm{IC}_{50}$ values of 49.65 and $1965 \mathrm{nM}$ were recorded for CPS and CPE, respectively (Fig. 2). The Z-factor for the assay was calculated to be 0.98 , indicating that the assay performed very well. Cryptolepis sanguinolenta however, demonstrated more potent activity against the late stage gametocytes compared to its major alkaloid, cryptolepine. The reference standard, dihydroartemisinin $\left(10-1.69 \times 10^{-4} \mu \mathrm{M}\right)$ exhibited an $\mathrm{IC}_{50}$ of $15 \mathrm{nM}$ (Fig. 2) falling within the range normally expected for the resazurin-based assay and other gametocytocidal assay platforms, as described by Reader et al. [11].

Taken together, C. sanguinolenta (CPS) and it major alkaloid, cryptolepine (CPE) have potent inhibitory effects on the late stage gametocyte of $P$. falciparum strain NF54 (Fig. 2).

\section{In vitro antiplasmodial interaction}

The in vitro $\mathrm{IC}_{50} \mathrm{~s}$ of cryptolepine and the standard antimalarial drugs in the asexual stages of $P$. falciparum 3D7 are presented in Table 1. In the in vitro interaction assays, cryptolepine's combination with chloroquine or lumefantrine showed additivity with a mean $\Sigma F I C_{50}$ of $1.342 \pm 0.34$ and $1.017 \pm 0.45$, respectively (Table 2 ). Cryptolepine combination with amodiaquine at all therapeutically relevant ratios showed a mean $\Sigma F I C_{50}$ $(0.235 \pm 0.15)$ of less than 0.8 suggesting synergism. Antagonism (mean $\Sigma \mathrm{FIC}_{50}=4.182 \pm 0.68$ ) was observed when cryptolepine was combined with mefloquine. Isobolograms indicating various cryptolepine-based combinations are presented in Fig. 3.

\section{Discussion}

The aqueous root extract of $C$. sanguinolenta has gained wide usage as an anti-malarial agent in both herbal and orthodox hospitals in Ghana and other West African countries. This coupled with the plant's established efficacy against the asexual stages of $P$. falciparum $[7,17]$ motivated this study to evaluate possible gametocytocidal properties of the plant and its major alkaloid, cryptolepine. In the current study, the stage-specific in vitro gametocytocidal activities of C. sanguinolenta (CPS) and its major alkaloid cryptolepine (CPE) were tested against the late-stage NF54 strain of the malaria parasite,
P. falciparum. The assay assesses gametocyte survival after drug exposure using resazurin-based assay, and indicated a potent loss of viability of the late stage gametocyte with both C. sanguinolenta (49.65 nM) and cryptolepine (1965 nM). This result is not surprising as earlier work [18] demonstrated potent in vitro loss of viability of the late stage gametocytes by local polyherbal antimalarial products containing C. sanguinolenta. Despite the demonstrated activity, the major alkaloid from the plant known to possess the most efficacious in vitro antiplasmodial activity showed less gametocytocidal activity compared to the root extract of the plant. This indicates the presence of other alkaloids in the aqueous root extract of the plant contributing to the high activity against gametocytes. Given the demonstrated high gametocytocidal properties of CPS and CPE, their prominent antiprotozoal activity may be attributed mainly to their effect on both the asexual and sexual stages of the Plasmodium parasite.

Our current study also sheds light on the in vitro antimalarial interaction of cryptolepine with four standard anti-malarial agents. The steady rise in the resistance of P. falciparum to current anti-malarial drugs, has directed current therapeutic strategies toward the development of combination therapies. Cryptolepine is a promising antimalarial compound effective against both chloroquinesensitive and chloroquine-resistant P. falciparum [9]. It has however been shown to possess DNA intercalating and topoisomerase II inhibitory effect $[19,20]$. This has directed research into structural modification of the compound to synthesize relatively safer derivatives. Previous pharmacokinetic reports of cryptolepine showed a rapid disappearance from the plasma and localization in various tissues except the CNS with possible hepatobiliary clearance pathway [21]. Aldehyde oxidase has been shown to be involved in the metabolism of cryptolepine [22]. Nanoformulation of cryptolepine hydrochloride demonstrated a better in vivo antiplasmodial chemosuppression, superior bioavailability and an increased half-life compared with the free compound [23]. A candidate drug partner is sought for the parallel development of cryptolepine, comparable to ACT encouraged by the WHO. This approach will provide cryptolepine with potential drug partners for further development to reduce the risk of drug resistance to $P$. falciparum. A new anti-malarial combination should preferably target both the rapidly replicating asexual stages and the less metabolically active, nonreplicating mature gametocytes.

The in vitro anti-malarial interaction of cryptolepine using the SYBR Green fluorescent assay demonstrated varied interaction with these standard agents. 

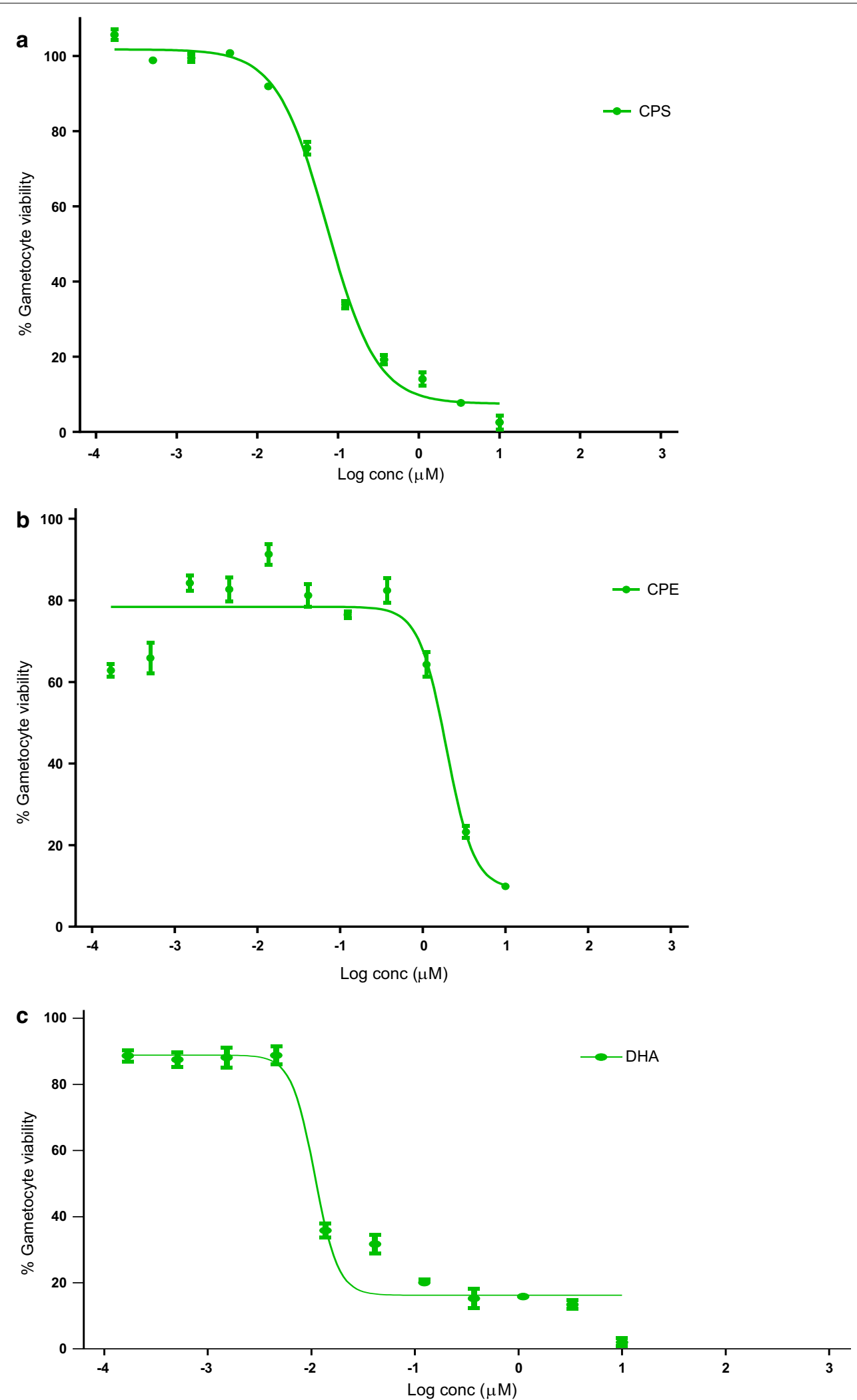

Fig. 2 Gametocyte viability of CPS and CPE. Gametocytocidal activity of CPS (a), CPE (b) and DHA (c) against late stage gametocytes. Bars represent mean gametocyte activity at each compound concentration (with standard deviation [SD]) 
Table 1 In vitro anti-malarial activity against asexual blood stages

\begin{tabular}{lc}
\hline Drugs & Mean IC $_{\mathbf{5 0}} \pm$ S.E.M (nM) \\
\hline Amodiaquine & $12.63 \pm 1.66$ \\
Mefloquine & $12.98 \pm 0.47$ \\
Lumefantrine & $10.80 \pm 0.97$ \\
Chloroquine & $11.05 \pm 1.79$ \\
Cryptolepine & $603.82 \pm 75.57$ \\
\hline
\end{tabular}

$\mathrm{IC}_{50}$ values of cryptolepine, lumefantrine, mefloquine, and chloroquine against Plasmodium falciparum strain 3D7 are expressed as mean \pm standard error of mean of the results of at least three independent assays

Cryptolepine showed varied interaction with the 4-aminoquinolines, amodiaquine and chloroquine. The combination of cryptolepine with amodiaquine had a synergistic effect in vitro (mean $\Sigma F I C=0.235 \pm 0.15$ ) whereas an additive effect (mean $\Sigma F I C=1.342 \pm 0.34$ ) was seen with chloroquine. The 4-aminoquinolines are considered to share, in principle, the same mode of action. However, a different interactive profile was found with cryptolepine. It is therefore wrong to equate amodiaquine to chloroquine in terms of activity. The synergy observed with amodiaquine may be due to the Mannich base structure in amodiaquine. A similar synergistic effect attributed to the Mannich base side chain in pyronaridine and amodiaquine has been reported with artemisinin [24]. An antagonistic effect was observed when cryptolepine was combined with mefloquine. Mefloquine has been shown to inhibit the uptake of chloroquine as well as chloroquine's ability to cause the accumulation of undigested haemoglobin [25].

Mefloquine (and possibly quinine) has also been hypothesized to inhibit endocytosis of the erythrocyte cytosol by the parasite, resulting in a lowered free haem concentration, to which chloroquine binds, in the digestive vacuole [25]. Cryptolepine, an indoloquinoline has been shown to act similarly to chloroquine by inhibiting the biomineralization of the toxic waste material haem into an insoluble complex, haemozoin, leading to membrane damage and parasite death [9, 26, 27]. On this background it is not surprising that antagonism was observed when cryptolepine was combined with mefloquine, possibly following a similar pathway of antagonism demonstrated by mefloquine when combined with chloroquine. In the case of cryptolepine-lumefantrine combination at therapeutically relevant concentration ratios, an additivity effect (mean $\Sigma F I C=1.017 \pm 0.45$ ) was observed.

The combination of cryptolepine with amodiaquine showed synergy in vitro in P. falciparum strain 3D7 and this combination provides a dual action with both agents inhibiting haemoglobin digestion in the asexual blood stages and amodiaquine inhibiting gametocyte maturation/gamete exflagellation by different mechanisms [28]. Such combinations with dual action are relevant in malaria endemic regions, where infections are usually asymptomatic with clinical symptoms developing late in the course of the disease, permitting the maturation of gametocytes and hence disease transmission [29, 30]. The enhanced anti-malarial activity of cryptolepine with amodiaquine may possibly result in low-dose treatment regimens and hence reduce toxicity. However, it should be noted that these assay results may vary in different plasmodium strains and further investigations is recommended in the early stages of gametocytes and in several $P$. falciparum strains and/or rodent malaria models to accentuate the interactions established.

\section{Conclusions}

Cryptolepis sanguinolenta and its major alkaloid, cryptolepine exhibited high activities against the late-stage gametocyte of $P$. falciparum NF54, attributing their primary targets as the mature gametocytes and intraerythrocytic parasite. Lumefantrine and chloroquine both showed additivity when each drug was combined with cryptolepine whereas antagonism was observed when cryptolepine was combined with mefloquine at therapeutically relevant concentration ratios. Cryptolepine in combination with the Mannich base, amodiaquine,

Table 2 In vitro interaction of cryptolepine combinations against 3D7 P. falciparum strains

\begin{tabular}{|c|c|c|c|c|c|c|}
\hline \multirow[t]{2}{*}{ Drug combination } & \multicolumn{4}{|l|}{$\Sigma \mathrm{FIC}_{50}$} & \multirow[t]{2}{*}{ Mean $\Sigma \mathrm{FIC}_{50}$} & \multirow[t]{2}{*}{ Interaction } \\
\hline & $4: 1$ & $3: 2$ & $2: 3$ & $1: 4$ & & \\
\hline Cryptolepine + amodiaquine & $0.640 \pm 0.08$ & $0.0234 \pm 0.11$ & $0.0631 \pm 0.07$ & $0.421 \pm 0.12$ & $0.287 \pm 0.10$ & Synergism \\
\hline Cryptolepine + mefloquine & $3.015 \pm 0.91$ & $3.013 \pm 1.01$ & $5.106 \pm 1.13$ & $5.595 \pm 0.91$ & $4.182 \pm 0.99$ & Antagonism \\
\hline Cryptolepine + lumefantrine & $2.1108 \pm 0.17$ & $1.6865 \pm 0.01$ & $0.1475 \pm 0.02$ & $0.1245 \pm 0.04$ & $1.017 \pm 0.06$ & Additivity \\
\hline Cryptolepine + chloroquine & $1.95 \pm 0.21$ & $1.775 \pm 0.36$ & $1.66 \pm 0.04$ & $0.475 \pm 0.05$ & $1.465 \pm 0.17$ & Additivity \\
\hline
\end{tabular}

The ratios 4:1, 3:2, 2:3 and 1:4 refer to fixed dosage ratios for drug A (cryptolepine) to drug $B$ (amodiaquine, mefloquine, lumefantrine, chloroquine). Values are the means from $\geq 3$ experiments 

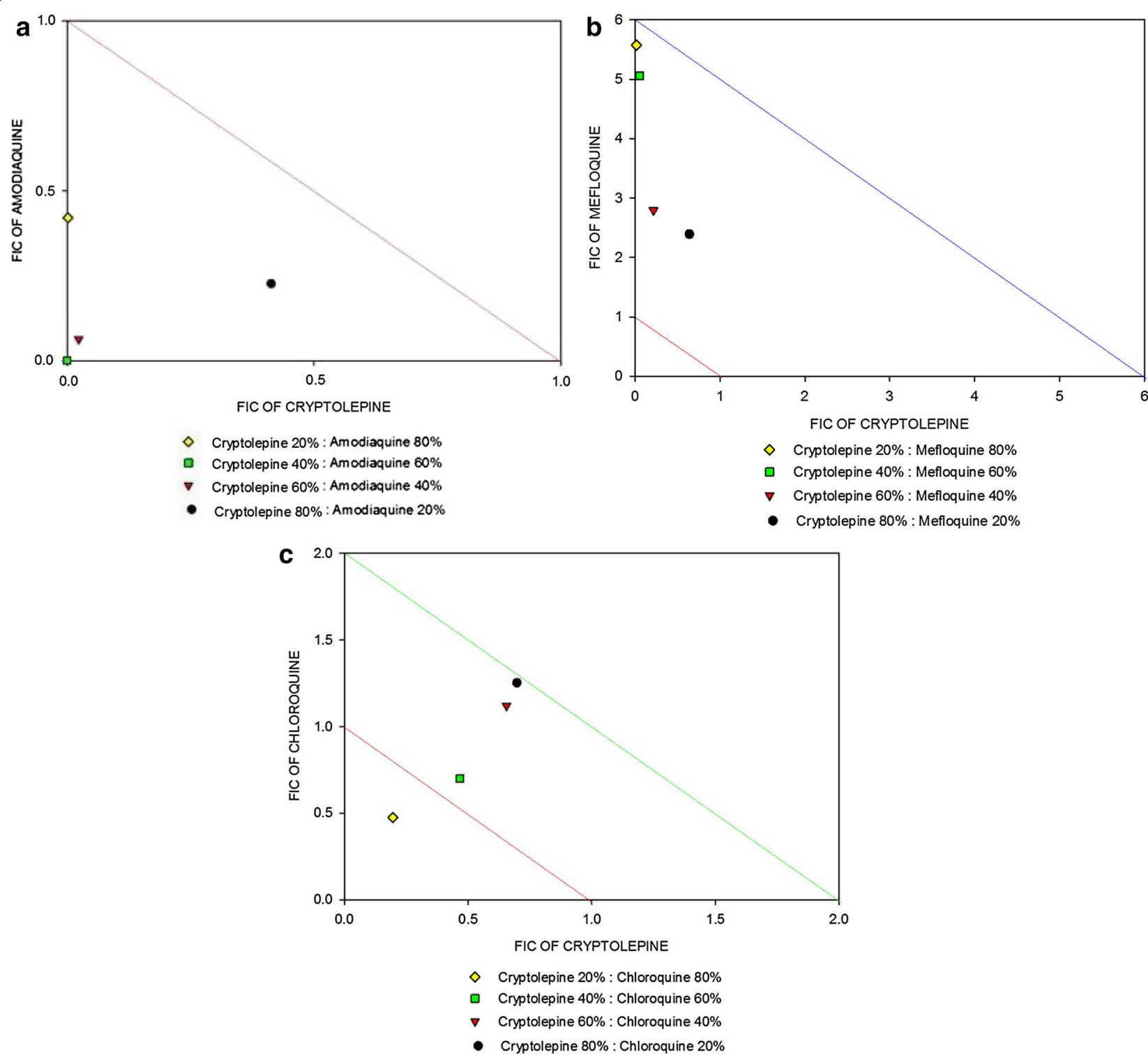

Fig. 3 Effects of combinations of cryptolepine with standard anti-malarial drugs on Plasmodium falciparum growth in vitro (3D7 strain). Isobolograms illustrating the effect of combinations of cryptolepine with amodiaquine (a), mefloquine $(\mathbf{b})$, and chloroquine (c). The interaction between cryptolepine and amodiaquine, mefloquine, chloroquine or lumefantrine against ring stage parasites was determined using the SYBR Green I fluorescence-based drug sensitivity assay with the fixed ratio method. Each combination was set up in triplicate for $48 \mathrm{~h}$. The FIC ${ }_{50}$ concentrations were used for plotting the isobologram

showed synergism. On this basis, the cryptolepineamodiaquine combination certainly deserves attention for further studies in regards to the synergy demonstrated.

\section{Abbreviations}

FIC: fractional inhibitory concentrations; Mean $\Sigma$ FICs: mean sums of fractional inhibitory concentrations; IC50: 50\% inhibitory concentration; WHO: World Health Organization; ACT: artemisinin-based combination therapy; CPE: cryptolepine; CPS: aqueous root extract of Cryptolepis sanquinolenta; LUM: lumefantrine; AMQ: amodiaquine; CQ: chloroquine; $\mathrm{nM}$ : nano-molar; $\mathrm{mM}$ : milli-molar; CPM: complete parasite medium; RBC: red blood cell; MSF: malaria SYBR Green I fluorescence.

\section{Authors' contributions}

ADF, DM, AT participated in the study design, carried out the experiments, performed the statistical analysis, and drafted the manuscript; CA, KBM, and BG participated in the study design and the execution of the experiments; ADF conducted the in vitro anti-malarial interaction assays. CW isolated cryptolepine hydrochloride from the plant, Cryptolepis sanguinolenta; KA, CA, CW, KBM and $B G$ contributed to the study design and revision of the manuscript. All authors read and approved the final manuscript. 


\begin{abstract}
Author details
1 Department of Pharmacology, Faculty of Pharmacy and Pharmaceutical Sciences, College of Health Sciences, Kwame Nkrumah University of Science and Technology, Kumasi, Ghana. ${ }^{2}$ Department of Immunology, Noguchi Memorial Institute for Biomedical Research, University of Ghana, Legon, Ghana. ${ }^{3}$ Biosciences, Council for Scientific and Industrial Research, P.O. Box 395, Pretoria 0001, South Africa. ${ }^{4}$ School of Pharmacy, University of Bradford, West Yorkshire BD7 1DP, UK.
\end{abstract}

\section{Competing interests}

The authors declare that they have no competing interests with the trademarks stated in this study. There is no financial gain or any other benefits from the cited trademarks.

\section{Availability of data and materials}

The datasets supporting the conclusions of this article are included within the article

\section{Consent for publication}

Not applicable.

\section{Ethics approval and consent to participate}

Not applicable.

\section{Publisher's Note}

Springer Nature remains neutral with regard to jurisdictional claims in published maps and institutional affiliations.

Received: 8 May 2017 Accepted: 18 December 2017

Published online: 28 December 2017

\section{References}

1. WHO. World malaria report, 2015. Geneva: World Health Organization; 2015.

2. Fidock DA. Drug discovery: priming the antimalarial pipeline. Nature. 2010;465:297-8

3. Wells TNC, Alonso PL, Gutteridge WE. New medicines to improve control and contribute to the eradication of malaria. Nat Rev Drug Discov. 2009:8:879-91.

4. Cheeseman IH, Miller BA, Nair S, Nkhoma S, Tan A, Tan JC, et al. A major genome region underlying artemisinin resistance in malaria. Science. 2012;336:79-82.

5. Dondorp AM, Nosten F, Yi P, Das D, Phyo AP, Tarning J, et al. Artemisinin resistance in Plasmodium falciparum malaria. N Engl I Med. 2009;361:455-67.

6. Boye GL, Ampofo O. Clinical uses of Cryptolepis sanquinolenta. In: Proceedings of the first international seminar on cryptolepine, 27-30 July 1983, University of Science and Technology, Kumasi, Ghana, p. 37-40.

7. Bugyei KA, Boye GL, Addy ME. Clinical efficacy of a tea-bag formulation of Cryptolepis sanguinolenta root in the treatment of acute uncomplicated falciparum malaria. Ghana Med J. 2010;44:3-9.

8. Cimanga K, De Bruyne T, Pieters L, Vlietinck AJ, Turger CA. In vitro and in vivo antiplasmodial activity of cryptolepine and related alkaloids from Cryptolepis sanguinolenta. J Nat Prod. 1997;60:688-91.

9. Wright CW, Addae-Kyereme J, Breen AG, Brown JE, Cox MF, Croft SL, et al. Synthesis and evaluation of cryptolepine analogues for their potential as new antimalarial agents. J Med Chem. 2001;44:3187-94.

10. Kuntworbe N, Martini N, Brimble M, Alany GR, Al-Kassas R. Isolation and development of an HPLC method for the detection and quantification of cryptolepine and its application in the determination of the total cryptolepine content in the root powder of Cryptolepis sanguinolenta. Pharmacol Pharm. 2012;3:263-70.
11. Reader J, Botha M, Theron A, Lauterbach SB, Rossouw C, Engelbrecht $D$, et al. Nowhere to hide: interrogating different metabolic parameters of Plasmodium falciparum gametocytes in a transmission blocking drug discovery pipeline towards malaria elimination. Malar J. 2015;14:213.

12. Tanaka TQ, Dehdashti SJ, Nguyen DT, MCKew JC, Zheng W, Williamson KC. A quantitative high throughput assay for identifying gametocytocidal compounds. Mol Biochem Parasitol. 2013;188:20-5.

13. Trager W, Jensen JB. Human malaria parasites in continuous culture. Science. 1976;193:673-5.

14. Fivelman QL, Adagu IS, Warhurst DC. Modified fixed-ratio isobologram method for studying in vitro interactions between atovaquone and proguanil or dihydroartemisinin against drug-resistant strains of Plasmodium falciparum. Antimicrob Agents Chemother. 2004;48:4097-102.

15. Zhang $\mathrm{JH}$, Chung TD, Oldenburg KR. A simple statistical parameter for use in evaluation and validation of high throughput screening assays. J Biomol Screen. 1999;4:67-73.

16. Snyder C, Chollet J, Wna Santo-Tomas J, Scheurer C, Wittlin S. In vitro and in vivo interaction of synthetic peroxide RBx11160 (OZ277) with piperaquine in Plasmodium models. Exp Parasitol. 2007;115:296-300.

17. Boye GL. Studies on antimalarial action of Cryptolepis sanguinolenta extract. In: Proceedings of the international symposium on east-west medicine, Seoul, Korea. 1989 October 10-11; p. 243-51.

18. Amoah LE, Kakaney C, Kwansa-Bentum B, Kusi KA. Activity of herbal medicines on Plasmodium falciparum gametocytes: implications for malaria transmission in Ghana. PLoS ONE. 2015;10:e0142587.

19. Ansah C, Gooderham NJ. The popular herbal antimalarial, extract of Cryptolepis sanguinolenta, is potently cytotoxic. Toxicol Sci. 2002;70:245-51.

20. Kirby GC, Paine A, Warhurst DC, Noamese BK, Phillipson JD. In vitro and in vivo antimalarial activity of cryptolepine, a plant-derived indoloquinoline. Phytother Res. 1995;9:359-63.

21. Salako Q, Ablordeppey SY, Dwuma-Badu D, Thornback FR. Radioiodination and preliminary in vivo investigation of the alkaloid cryptolepine. Int J Appl Radiat Isot. 1985;36:1003-4.

22. Stell JGP, Wheelhouse RT, Wright CW. Metabolism of cryptolepine and 2-fluorocryptolepine by aldehyde oxidase. J Pharm Pharmacol. 2012;64:237-43.

23. Kuntworbe $\mathrm{N}$, Ofori M, Addo P, Tingle M, Al-Kassas R. Pharmacokinetics and in vivo chemosuppressive activity studies on cryptolepine hydrochloride and cryptolepine-loaded gelatine nanoformulation designed for parenteral administration for the treatment of malaria. Acta Trop. 2013;127:165-73

24. Pradines B, Tall A, Parzy D, Spiegel A, Fusai T, Hienne R, et al. In vitro activity of pyronaridine and amodiaquine against African isolates (Senegal) of Plasmodium falciparum in comparison with standard antimalarial drugsMannich bases: promising drugs for Africa? J Antimicrob Chemother. 1998:42:333-9.

25. Famin $\mathrm{O}$, Ginsburg $\mathrm{H}$. Differential effects of 4-aminoquinoline-containing antimalarial drugs on haemoglobin digestion in Plasmodium falciparuminfected erythrocytes. Biochem Pharmacol. 2002;63:393-8.

26. Foley M, Tilley L. Quinoline antimalarials: mechanisms of action and resistance and prospects for new agents. Pharmacol Ther. 1998;79:55-87.

27. Kumar S, Guha M, Choubey V, Maity P, Bandyopadhyay U. Antimalarial drugs inhibiting hemozoin ( $\beta$-hematin) formation: a mechanistic update. Life Sci. 2007;80:813-28.

28. Delves M, Plouffe D, Scheurer C, Meister S, Wittlin S, Winzeler EA, et al. The activities of current antimalarial drugs on the life cycle stages of Plasmodium: a comparative study with human and rodent parasites. PLoS Med. 2012;9:e1001169.

29. Greenwood BM. Asymptomatic malaria infections-do they matter? Parasitol Today. 1987;3:206-14.

30. Stepniewska K, Price RN, Sutherland CJ, Drakeley CJ, von Seidlein L, Nosten F, et al. Plasmodium falciparum gametocyte dynamics in areas of different malaria endemicity. Malar J. 2008;7:249. 